\title{
Resonant Escape over an Oscillating Barrier in Underdamped Josephson Tunnel Junctions
}

\author{
Yang Yu* and Siyuan Han \\ Department of Physics and Astronomy, University of Kansas, Lawrence, Kansas 66045, USA
}

(Received 2 May 2003; published 18 September 2003)

\begin{abstract}
The escape from a metastable state over an oscillating barrier of an underdamped Josephson tunnel junction has been experimentally investigated with oscillation frequency well separated from the plasma frequency of the junction. The resonant escape, namely, a minimum of the average escape time as a function of the oscillation frequency, was observed. For the oscillation frequency much smaller than the "resonant frequency," the average escape time is the average of the times required to cross over each of the barriers. On the other hand, for the oscillation frequency much greater than the "resonant frequency," the average escape time is that required to cross the average barrier.
\end{abstract}

The thermal activated escape of a particle of mass $m$ over a potential barrier $\Delta U$ is a ubiquitous problem in physics, chemistry, and biology [1,2]. The escape rate caused by thermal fluctuation at temperature $T$ is given by the famous Kramers rate,

$$
\Gamma=\left(a_{t} \omega_{0} / 2 \pi\right) \exp \left(-\Delta U / k_{B} T\right),
$$

with $\omega_{0}^{2} \equiv U^{\prime \prime} / m$ being the natural frequency of the system at the minima of the potential $U$, and $k_{B}$ being Boltzmann's constant. The prefactor $a_{t}$ is usually smaller than unity, and weakly dependent on the damping of the system [2]. In general, Eq. (1) is only valid in the situation $\Delta U \gg k_{B} T$. Therefore $\Gamma$ is much smaller than $\omega_{0}$. If we apply a weak periodic force of frequency $f$ to the particle, the particle can be resonantly activated out of the potential well at $f \approx \omega_{0}$ [3]. For $f \ll \omega_{0}$, another interesting phenomenon called stochastic resonance has been observed for a double-well potential at $f \approx \Gamma$ [4]. Recently, it was shown theoretically that when the potential barrier itself is fluctuating with a frequency $f \ll \omega_{0}$, a counterintuitive resonant behavior might occur [5]. In the limits of very slow and fast fluctuation, the average escape times are the average of the crossing times over each of the barriers and the crossing time of the average barrier, respectively. At intermediate rates, the crossing is strongly correlated with the potential fluctuations and the average escape time exhibits a minimum at a resonant fluctuation rate. The original work of Doering and Gadoua triggered a large amount of theoretical analysis and simulations [6-17]. For example, the resonant escape was also predicted in the periodically oscillating barrier $[16,18]$. However, the experimental investigation has been sparse so far. The observation of resonant escape was reported only in a circuit model [19]. In addition, all the works for $f \ll \omega_{0}$ were focused on the overdamped systems. Whether the resonant escape can be observed in underdamped systems remains as an open question due to the challenge of an analytical treatment. In this Letter, we report the experimental observation of the resonant escape in a real underdamped physical system: Josephson tunnel junctions (JTJ).

It is well established that a current-biased JTJ is an excellent system to study the dynamics of a Brownian particle in a metastable potential [20]. The equation of motion of a current-biased JTJ, $C d^{2} \Phi / d t^{2}+$ $R^{-1} d \Phi / d t=-\partial U / \partial \Phi$, is identical to the classical equation of motion of a particle of mass $C$ moving in a washboard potential $U(\Phi)=-I_{b} \Phi-E_{J} \cos \left(2 \pi \Phi / \Phi_{0}\right)$, where $C$ is the junction capacitance, $R$ is the shunt resistance, $I_{b}$ is the bias current, $E_{J} \equiv I_{c} \Phi_{0} / 2 \pi$ is the Josephson coupling energy, $I_{c}$ is the critical current of the junction, $\Phi_{0} \equiv h / 2 e$ is the flux quantum, and $\Phi$ is the phase difference across the junction measured in units of $\Phi_{0} / 2 \pi$ (see Fig. 1). For $I_{b}<I_{c}$ the potential displays a series of metastable wells with barrier height

$$
\Delta U=2 E_{J}\left(\sqrt{1-i^{2}}-i \cos ^{-1} i\right),
$$

where $i \equiv I_{b} / I_{c}$ is the normalized bias current. A junction initially trapped in a zero-voltage state (the particle localized in one of the wells) can be activated out of the potential well by thermal fluctuations. The lifetime of the zero-voltage state is obtained from Eq. (1) [21]
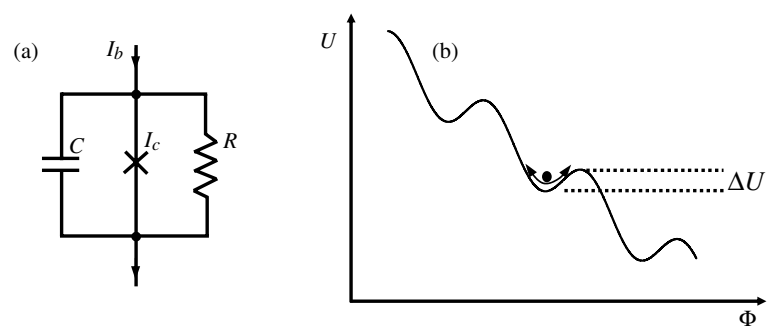

FIG. 1. Equivalent circuit of a current-biased JTJ (a), and $U$ vs $\Phi$ for a bias current $I_{b}=0.5 I_{c}$ (b). In order to show the potential well clearly, we chose a smaller bias current than those used in the experiments. 


$$
\tau \equiv 1 / \Gamma=\left(a_{t} \frac{\omega_{p}}{2 \pi}\right)^{-1} \exp \left(\Delta U / k_{B} T\right)
$$

with $\omega_{0}$ replaced by $\omega_{p}=\sqrt{2 \pi I_{c} / \Phi_{0}} \bar{C}\left(1-i^{2}\right)^{1 / 4}$, the oscillation frequency of the particle at the bottom of the well. In the low to extremely low damping regime, relevant for our experiment, $a_{t}$ is given by $a_{t}=$ $4 /\left[\sqrt{1+\left(Q k_{B} T / 1.8 \Delta U\right)}+1\right]^{2}[22]$, here $Q$ is the quality factor defined as $Q=\omega_{p} R C$. For an underdamped junction, after escape from the potential well, the particle can run freely down the washboard, corresponding to a finitevoltage state of the junction.

The samples used in this study are $\mathrm{NbN} / \mathrm{AlN} / \mathrm{NbN}$ JTJs. The critical temperature $T_{c}$ of the junctions was $\sim 16 \mathrm{~K}$. The junctions were of high quality, with a sharp rise of the quasiparticle tunneling current at $2 \Delta_{s} / e$, where $\Delta_{s} \approx 2.7 \mathrm{meV}$ is the superconducting energy gap of $\mathrm{NbN}$. The data reported below were taken from a $10 \times$ $10 \mu \mathrm{m}^{2}$ junction on the chip so that parasitic capacitance of the on-chip leads had negligible effect on the junction [23]. The critical current and the capacitance of the junction, obtained from independent measurements in the thermal regime, were $I_{c} \simeq 150 \mu \mathrm{A}$ and $C \approx 5.8 \mathrm{pF}$. The sample was mounted on a chip carrier that was enclosed in a helium-filled oxygen-free copper sample cell, which was thermally anchored to the mixing chamber of a dilution refrigerator. The junction was magnetically shielded by two Mu-metal cylinders surrounding the inner vacuum can and outer vacuum can, respectively. All electrical leads that connected the junctions to room temperature electronics were carefully filtered by EMI filters (at $300 \mathrm{~K}$ ), RC filters (at $1.4 \mathrm{~K}$ ), and microwave filters based on lossy coaxial line (at $10 \mathrm{mK}$ ) [24]. Battery-powered low-noise preamplifiers were used for all measurements. The measured junction voltage noise spectrum showed no peak at $60 \mathrm{~Hz}$ or its harmonics, confirming the good quality of our experimental setup.

The escape times of the junction were directly measured using the time-domain technique. The more detailed description of the time-domain technique can be found elsewhere [25]. Briefly, for each escape event, we started the cycle by ramping up the bias current to a value $I_{b}\left(\sim 0.9 I_{c}\right)$ and maintaining at this level for a period of waiting time (typically $20 \mathrm{~ms}$ ). A square-wave oscillating current with amplitude $2 \Delta I_{b}\left(\Delta I_{b} \ll I_{b}\right)$, frequency $f$ can be added to the bias current during the waiting time. Therefore, the total bias current is a squarewave function oscillated between $I_{b}+\Delta I_{b}$ and $I_{b}-\Delta I_{b}$, resulting in a piecewise oscillation of the barrier height between $\Delta U_{-}$and $\Delta U_{+}$. The junction voltage was fed to a timer, which was triggered by the sudden voltage jump when the junction switched from the zero-voltage state to the finite-voltage state, to record escape time $t_{\mathrm{esc}}$. The bias current $I_{b}$ was then decreased to zero, returning the junction to the zero-voltage state. The process was repeated about $10^{4}$ times to obtain an ensemble of the escape time. In the absence of oscillating current, the lifetime $\tau$ at a constant bias current can be obtained from the escape time [25]. For the oscillating barrier, the average time of the ensemble represents the mean first-passage time $\left\langle t_{\text {esc }}\right\rangle$ [5].

Figure 2 shows examples of $\left\langle t_{\mathrm{esc}}\right\rangle$ as a function of oscillation frequency. The resonant escape is clearly observed. The lifetimes of the zero-voltage state at the configurations $\Delta U_{-}$and $\Delta U_{+}$were directly measured and noted as $\tau_{-}$and $\tau_{+}$, respectively. In the limit of low frequency $f \ll 1 / \tau_{+}$, the average escape time is the average lifetime of the system cross $\Delta U_{-}$and $\Delta U_{+},\left\langle t_{\mathrm{esc}}\right\rangle \simeq\left(\tau_{-}+\tau_{+}\right) / 2$. On the other hand, for the high oscillating frequency $f \gg 1 / \tau_{-}$, the average escape time is the lifetime of the system cross the average barrier $\left(\Delta U_{-}+\Delta U_{+}\right) / 2$. Using Eq. (3), $\left\langle t_{\mathrm{esc}}\right\rangle \simeq$ $\left(a_{t} \omega_{p} / 2 \pi\right)^{-1} e^{\left(\Delta U_{-}+\Delta U_{+}\right) / 2 k_{B} T}$, which is about $\sqrt{\tau_{-} \tau_{+}}$for the small oscillating amplitude $\Delta I_{b} \ll I_{b}$. The frequency at which the lifetime is a minimum, namely, resonant frequency $f_{\text {res }}$, is on the order of $1 / \tau_{-}$. The inset of Fig. 2 shows the good linear relation between $f_{\text {res }}$ and $1 / \tau_{-}$. These signatures of resonant escape are quantitatively consistent with that of the theoretical observation in an overdamped system $[5,16]$, which indicates that the underlying physical mechanism of the resonant escape is most likely the same and robust in underdamped systems. Also, since the oscillation frequency of the barrier is several orders of magnitude smaller than $\omega_{p}\left(\sim 10^{11} \mathrm{rad} / \mathrm{s}\right.$ estimated from the parameters of the

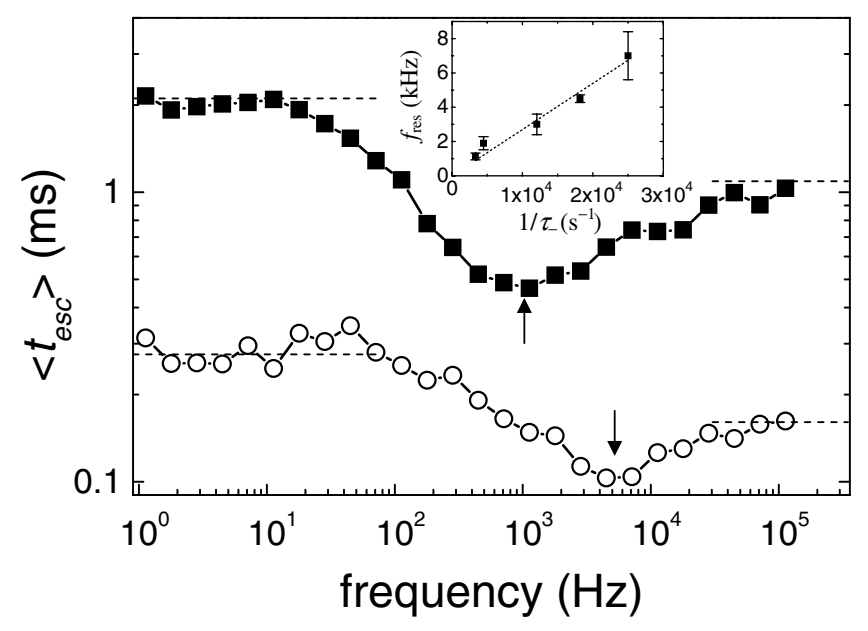

FIG. 2. Average escape time as a function of the oscillating frequency at $4.2 \mathrm{~K}$. The lifetimes directly measured using time-domain technique are as follows: $\tau_{-}=0.301 \pm 0.005 \mathrm{~ms}$ and $\tau_{+}=3.904 \pm 0.032 \mathrm{~ms}$ for the solid squares, $\tau_{-}=$ $0.055 \pm 0.005 \mathrm{~ms}$ and $\tau_{+}=0.485 \pm 0.002 \mathrm{~ms}$ for the open circles. The dashed lines indicated the $\left(\tau_{-}+\tau_{+}\right) / 2$ and $\sqrt{\tau_{-} \tau_{+}}$in both cases. The arrows show $f_{\text {res }}$. The solid lines are guides for the eye. Inset: the resonant frequency $f_{\text {res }}$ as a function of $1 / \tau_{-}$. The dotted line, $f_{\text {res }}=0.27 / \tau_{-}$, is the best fit. 
junction), one can exclude that the resonant escape originated from the periodic driving force with frequency matching the natural frequency of the system. Additionally, it is worth noting that the average escape time is longer than $1 \mathrm{~ms}$, which is much greater than the inverse of the small oscillation frequency. Therefore, the requirement of infrequent scapes, which is not always fulfilled in the simulation circuit, was fully satisfied [2].

In addition to changing with the barrier height $\Delta U$, the resonant escape also depends on the intensity of thermal fluctuation. The resonant escape was observed at temperatures down to $1.6 \mathrm{~K}$, which is well above classicalquantum cross temperature $T_{\mathrm{cr}} \equiv \hbar \omega_{p} / 2 \pi k_{B} \approx 0.3 \mathrm{~K}$ [26]. It was found that the resonant escape mainly relies on the $\tau_{-}$and $\tau_{+}$. Similar to that at $4.2 \mathrm{~K}$, the asymptote $\left\langle t_{\text {esc }}\right\rangle$ at low (high) frequency is $\left(\tau_{-}+\tau_{+}\right) / 2\left(\sqrt{\tau_{-}} \overline{\tau_{+}}\right)$, and the resonant frequency is proportional to $1 / \tau_{-}$. A critical comparison of the experimental data and the thermal activation theory can be obtained by scaling all the experimental result to one curve. From Eq. (3), we obtain the ratio of $\left\langle t_{\mathrm{esc}}\right\rangle$ in the two limits

$$
\begin{aligned}
R_{\left\langle t_{\mathrm{esc}}\right\rangle} & =\frac{\tau_{-}+\tau_{+}}{2 \sqrt{\tau_{-} \tau_{+}}} \simeq \frac{e^{\Delta U_{-} / k_{B} T}+e^{\Delta U_{+} / k_{B} T}}{2 e^{\left(\Delta U_{-}+\Delta U_{+}\right) / 2 k_{B} T}} \\
& =\cosh \left(\Delta U_{\mathrm{amp}} / 2 k_{B} T\right),
\end{aligned}
$$

where $\Delta U_{\text {amp }} \equiv \Delta U_{+}-\Delta U_{-}$is the amplitude of oscillating barrier. In Fig. 3 we plotted $R_{\left\langle t_{\mathrm{esc}}\right\rangle}$ vs $\Delta U_{\mathrm{amp}} / k_{B} T$ using the data measured at different temperatures. Considering there is no adjustable parameter, the agreement between the theoretical prediction and the experiment is substantial.

Finally, we investigated the survival probability at different oscillating frequencies. In the thermal regime,

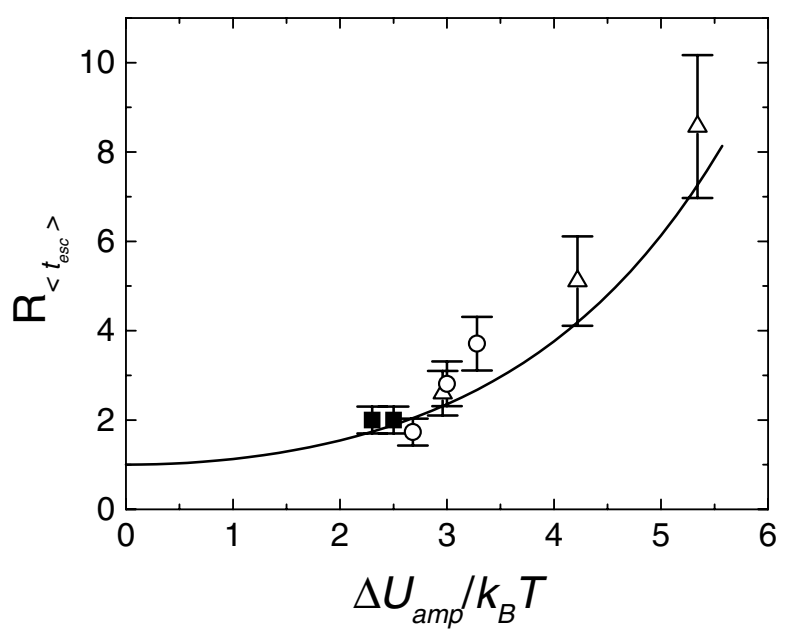

FIG. 3. $R_{\left\langle t_{\text {esc }}\right\rangle}$ vs $\Delta U_{\text {amp }} / k_{B} T$ of the data (symbols) and the prediction of thermal activation theory (line). The data were measured at 4.2 (solid squares), 3.8, 3.4, 3.1 (open circles from left to right), and $1.6 \mathrm{~K}$ (triangles). $\Delta U$ were calculated using Eq. (2). if a JTJ is prepared in the zero-voltage state at $t=0$, the probability of finding the junction remaining in this state [i.e., the survival probability $P_{s}(t)$ ] decays exponentially with a time constant (i.e., lifetime) $\tau$

$$
P_{s}(t)=1-P_{\mathrm{esc}}(t)=e^{-t / \tau}
$$

where $P_{\text {esc }}(t)$ is the escape probability. In our experiment, the escape probability density was obtained by making a histogram of the ensemble of escape times. Then the escape probability as a function of time $P_{\text {esc }}(t)$ was calculated by integrating the escape probability density. Shown in Fig. 4 are some of the examples of $P_{s}(t)$. When the oscillating frequency is much smaller than the resonant frequency $f_{\text {res }}$, the system escaped from two configurations with equal probability. Therefore, one can observe a two-constant decay of the survival probability. The total escape probability from $\Delta U_{-}$( or $\Delta U_{+}$) is $\sim 0.5$. But when the oscillation frequency is greater than $f_{\text {res }}$, there is only one decay constant, which suggests that the particle sees only one average barrier. For $f \gg f_{\text {res }}$, the lifetime obtained from the best fit is $\sim 1.12 \mathrm{~ms}$, which is the time of crossing the average barrier. For $f \simeq f_{\text {res }}$, the lifetime is $\sim 0.57 \mathrm{~ms}$. This value is about $2 \tau_{-}$, and agrees with the theoretical prediction for the overdamped system [16]. Therefore, the examination of the survival probability strongly suggests the similarity of the physical mechanism of the resonant escape in underdamped and overdamped systems.

In summary, we observed the resonant escape phenomena in an underdamped JTJ with oscillating barrier. It is interesting that in the thermal regime, the experimental results agreed quantitatively with the theoretical prediction in the overdamped system. Although further comparison is needed after the theory is developed to the

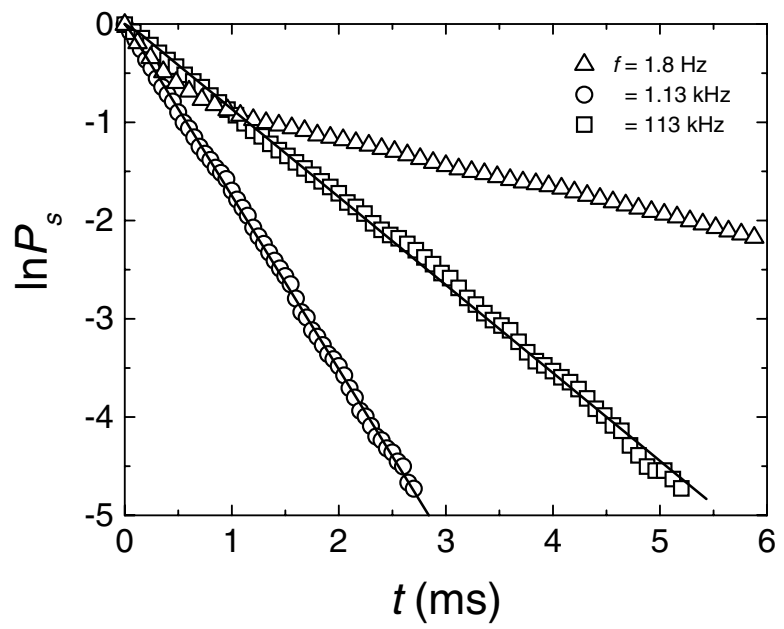

FIG. 4. Survival probability $P_{\mathrm{s}}(t)$ at different oscillating frequencies. The vertical axis is logarithmic. For slow oscillation, two-constant decay was observed, while for fast oscillation, only one-constant decay was observed. 
underdamped system, we believe that the physical mechanism is the same in both systems.

The authors are grateful to Dr. Zhen Wang for fabricating the samples. This work was supported in part by AFOSR (F49620-99-0205), the state of Kansas (S99041), and the NSF (DMR-9876874). Y.Y. was also supported by AFOSR (F49620-01-1-0457). One of the authors, Y. Y, thanks T. Orlando for fruitful discussions.

*Present address: Department of Electrical Engineering and Computer Science, MIT, Cambridge, Massachusetts 02139

[1] H. A. Kramers, Physica (Utrecht) 7, 284 (1940).

[2] P. Hänggi, P. Talkner, and M. Borkovec, Rev. Mod. Phys. 62, 251 (1990).

[3] M. H. Devoret, J. M. Martinis, D. Esteve, and J. Clarke, Phys. Rev. Lett. 53, 1260 (1984).

[4] For a review, see L. Gammaitoni, P. Hänggi, P. Jung, and F. Marchesoni, Rev. Mod. Phys. 70, 223 (1998).

[5] C. R. Doering and J. C. Gadoua, Phys. Rev. Lett. 69, 2318 (1992).

[6] U. Zürcher and C. R. Doering, Phys. Rev. E 47, 3862 (1993).

[7] C. Van den Broeck, Phys. Rev. E 47, 4579 (1993).

[8] M. Bier and R. D. Astumian, Phys. Rev. Lett. 71, 1649 (1993); Phys. Lett. A 247, 385 (1998).
[9] J. J. Brey and J. Casado-Pascual, Phys. Rev. E 50, 116 (1994).

[10] P. Pechukas and P. Hänggi, Phys. Rev. Lett. 73, 2772 (1994).

[11] A. J. R. Madureira et al., Phys. Rev. E 51, 3849 (1995).

[12] P. Reimann, Phys. Rev. Lett. 74, 4576 (1995); Phys. Rev. E 52, 1579 (1995).

[13] J. Iwaniszewski, Phys. Rev. E 54, 3173 (1996).

[14] M. Marchi et al., Phys. Rev. E 54, 3479 (1996).

[15] P. Reimann and T. C. Elston, Phys. Rev. Lett. 77, 5328 (1996).

[16] M. Boguñá et al., Phys. Rev. E 57, 3990 (1998).

[17] P. Reimann, R. Bartussek, and P. Hänggi, Chem. Phys. 235, 11 (1998).

[18] J. Maddox, Nature (London) 359, 771 (1992).

[19] R. N. Mantegna and B. Spagnolo, Phys. Rev. Lett. 84, 3025 (2000).

[20] W. C. Stewart, Appl. Phys. Lett. 12, 277 (1968); D. E. McCumber, J. Appl. Phys. 39, 3113 (1968).

[21] T. A. Fulton and L. N. Dunkleberger, Phys. Rev. B 9, 4760 (1974).

[22] M. Büttiker, E. P. Harris, and R. Landauer, Phys. Rev. B 28, 1268 (1983).

[23] J. P. Kauppinen and J. P. Pekola, Phys. Rev. Lett. 77, 3889 (1996).

[24] A. B. Zorin, Rev. Sci. Instrum. 66, 4296 (1995).

[25] S. Han et al., Science 293, 1457 (2001); Y. Yu et al., Supercond. Sci. Technol. 15, 555 (2002).

[26] I. Affleck, Phys. Rev. Lett. 46, 388 (1981). 